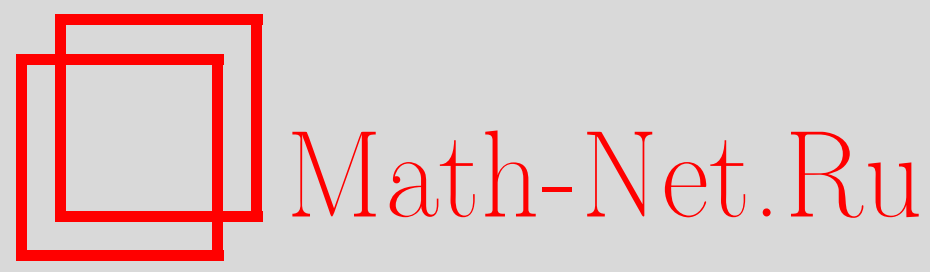

Н. В. Заболоцкий, Существование угловой плотности корней целых функций нулевого порядка, Матем. заметки, 2003, том 73, выпуск 5, 698-703

DOI: https://doi.org/10.4213/mzm225

Использование Общероссийского математического портала Math-Net.Ru подразумевает, что вы прочитали и согласны с пользовательским соглашением http://www.mathnet.ru/rus/agreement

Параметры загрузки:

IP: 34.229 .45 .116

26 апреля 2023 г., 12:50:09

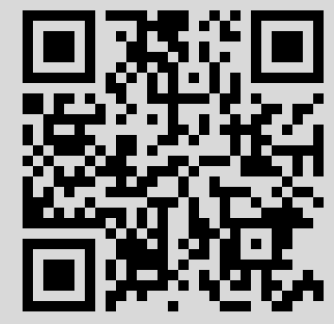




\section{СУЩЕСТВОВАНИЕ УГЛОВОЙ ПЛОТНОСТИ КОРНЕЙ ЦЕЛЫХ ФУНКЦИЙ НУЛЕВОГО ПОРЯДКА}

\section{Н. В. Заболоцкий}

Найдены достаточные условия при которых из силной регулярности роста целых функций нулевого порядка вытекает существование угловой плотности их корней.

Библиограффия: 8 названий.

Пусть $f$ - целая функция порядка $\rho, 0<\rho<\infty, f(0)=1, \rho(r)$ - ее уточненньй порядок, т.е.

a) $\rho(r)$ - непрерьвно дифференцируемая на $[0,+\infty)$ функция;

б) $\rho(r) \rightarrow \rho$ при $r \rightarrow \infty$;

в) $r \rho^{\prime}(r) \ln r \rightarrow 0$ при $r \rightarrow \infty$;

г) $0<\varlimsup_{r \rightarrow \infty} T(r, f) / V(r)<+\infty$, где $V(r)=r^{\rho(r)}$.

Следуя Б.Я. Левину [1, с. 183], будем говорить, что функция $f$ имеет вполне регулярный рост (BРP) на луче $\arg z=\theta$, если существует предел

$$
\lim _{r \rightarrow \infty} * \frac{\ln \left|f\left(r e^{i \theta}\right)\right|}{V(r)}=h_{f}(\theta),
$$

где $\lim ^{*}$ обозначает, что $r$ стремится к бесконечности вне некоторого $E^{0}$-множества. Под $E^{0}$-множеством понимаем множество $E \subset \mathbb{R}$ такое, что $\operatorname{mes}(E \cap[0, r]) \rightarrow 0$ при $r \rightarrow \infty$.

Функцию $f$ будем называть функиией ВРР, если она является функцией ВРР во всей плоскости, т.е. при $0 \leqslant \theta<2 \pi$.

Пусть $n(r, \alpha, \beta)$ - число корней $a_{j}$ функции $f$, лежащих в секторе $\{z:|z| \leqslant r, \alpha<$ $\arg z \leqslant \beta\}, 0 \leqslant \alpha<\beta<2 \pi$. Будем говорить [1, с. 118-119], что множество корней целой функции имеет угловую плотность, если величина

$$
\Delta(\alpha, \beta)=\lim _{r \rightarrow \infty} \frac{n(r, \alpha, \beta)}{V(r)}
$$

существует для всех $\alpha, \beta$ за исключением, быть может, $\alpha$ или $\beta$, принадлежащих некоторому счетному множеству.

В $[1$, гл. 2,3$]$ дано полное описание целых функций ВРР. В частности, если $\rho$ - нецелое число, то целая функция $f$ является функцией ВРР тогда и только тогда, когда множес-

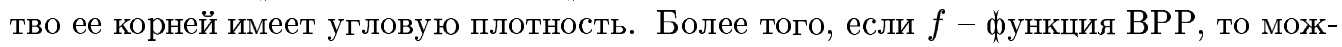
но установить асимптотическое поведение не только для функции $\ln \left|f\left(r e^{i \theta}\right)\right|$, но и для 
функции $\arg f(z)$, т.е. можно получить асимптотическое представление функции $\ln f(z)$. Здесь и далее $\ln f$ однозначная ветвь $\operatorname{Ln} f$ в области $D=\mathbb{C} \backslash \bigcup_{j=1}^{\infty}\left\{z:|z| \geqslant\left|a_{j}\right|, \arg z=\right.$ $\left.\arg a_{j}\right\}, \ln f(0)=0$.

Если для целых функций нулевого порядка аналогично ввести понятие ВРР, то [2, c. 72] $f$ является функцией ВРР тогда и только тогда, когда $f$ имеет ВРР хотя бы на одном луче. Более того, тогда

$$
h_{f}(\theta)=\lim _{r \rightarrow \infty} * \frac{\ln \left|f\left(r e^{i \theta}\right)\right|}{V(r)}=\lim _{r \rightarrow \infty} \frac{N(r)}{V(r)}=K,
$$

где

$$
0<K<+\infty, \quad N(r)=\int_{1}^{r} \frac{n(t, 0,2 \pi)}{t} d t .
$$

Отсюда видим, что ВРР целой функции нулевого порядка не зависит от аргументов ее корней, а только от их модулей. Заметим [3, c. 228], что тогда величина $\Delta(\alpha, \beta)=0$ для всех $\alpha$ и $\beta, 0<\alpha<\beta \leqslant 2 \pi$.

В [4] введено новое понятие регулярности роста для целых функций нулевого порядка, которое обладает свойствами, похожими на те, которьми обладает понятие ВРР цельх функций конечного ненулевого порядка.

Пусть $f$ - целая функция нулевого порядка, $f(0)=1$. Через $\lambda(r)$ обозначим уточненный порядок функции $n(r)=n(r, 0,2 \pi)$, т.е. функция $\lambda(r)$ удовлетворяет условиям а)-в) уточненного порядка с $\rho=0$, и, кроме того:

г) функция $v(r)=r^{\lambda(r)}$ не убывает и стремится к $+\infty$ при $r \rightarrow+\infty$;

д) $0<\varlimsup_{r \rightarrow \infty} n(r) / v(r)<+\infty$.

Не уменьшая общности, будем считать, что $v(r)=0$ и $n(r)=0$ для $0 \leqslant r \leqslant 1$. Назовем обыкновенны.ми те лучи $\arg z=\theta$, которые удовлетворяют условию

$$
\lim _{h \rightarrow 0}\left\{\varlimsup_{r \rightarrow \infty} \frac{n(r, \theta-h, \theta+h)}{v(r)}\right\}=0 .
$$

Все другие лучи мы будем называть исключительны.ми. Из монотонности функции $\Delta(\theta)$ легко заключить, что если корни функции $f$ имеют угловую плотность, то множество исключительньх лучей не более чем счетно.

Определение угловой плотности корней целой функции $f$ нулевого порядка вводим аналогично по формуле $(1)$, где лиш вместо $V(r)$ ставим $v(r)$.

Будем говорить, что целая функция $f$ нулевого порядка имеет на обькновенном луче $\arg z=\theta$ сильно регулярный рост (CРP), если существует предел

$$
H_{f}(\theta)=\lim _{r \rightarrow \infty} \frac{\ln f\left(r e^{i \theta}\right)-N(r)}{v(r)} .
$$

Функция $f$ назьвается функиией СРР на некотором множестве $R_{M}, M \subset[0,2 \pi)$, обыкновенных лучей, если величина $H_{f}(r, \theta)=\left(\ln f\left(r e^{i \theta}\right)-N(r)\right) / v(r)$ стремится к $H_{f}(\theta)$ при $\theta \in M, r \rightarrow \infty, r \notin E_{M}, E_{M} \subset E^{0}$. При этом на любом замкнутом подмножестве $M_{0}$ множества $M$ стремление переменной $H_{f}(r, \theta)$ к пределу $H_{f}(\theta)$ равномерно относительно $\theta$.

Если целая функция $f$ нулевого порядка имеет СРP на всех обькновенных лучах $\arg z=\theta,-\pi \leqslant \theta<\pi$, то $f$ будем называть функиией СРР. 
В [4] показано, если $f$ - целая функция нулевого порядка, множество корней которой имеет угловую плотность относительно функции $v(r)$, то функция $f$ имеет СРР. Наоборот, пусть $f$ - целая функция нулевого порядка СРР, нули которой находятся на конечном числе лучей. Тогда множество ее корней имеет угловую плотность.

Условие размещения корней на конечной системе лучей в последнем утверждении существенно. В общем случае из СРР целой функции, вообще говоря, не следует существование угловой плотности ее корней. Так в [5] доказано следующее утверждение.

Теорема А. Пусть $\alpha(r)$ - непрерывная положительная возрастающая $\kappa+\infty$ на $[0,+\infty)$ функиия. Тогда для произвольной возрастающей $\kappa+\infty$ на $[0,+\infty)$ функиии $\beta(r)$ существуют иелая функиия $f$ нулевого порядка и $E^{0}$-множество $E$ такие, что для почти всех $\theta \in[0,2 \pi)$ выполняется

$$
\ln f\left(r e^{i \theta}\right)=N(r)+o(\beta(r)), \quad r \rightarrow \infty, \quad r \notin E, \quad \varlimsup_{r \rightarrow \infty} \frac{n(r)}{\alpha(r)}=1
$$

и корни $f$ не имеют угловой плотности относительно функции $\alpha(r)$.

ЗАмЕчАнИЕ. Пусть $f$ - целая функция из теоремы А, $\lambda(r)$ - уточненный порядок считающей функции ее корней. Положив $\alpha(r)=v(r)$ из теоремы А получаем, что для почти всех $\theta, 0 \leqslant \theta<2 \pi$,

$$
\lim _{r \rightarrow \infty} \frac{\ln f\left(r e^{i \theta}\right)-N(r)}{v(r)}=0
$$

а корни $f$ не имеют угловой плотности. Заметим, что для функции $f$ предел

$$
\lim _{r \rightarrow \infty} \frac{N(r)}{v_{0}(r)}
$$

не существует, где

$$
v_{0}(r)=\int_{0}^{r} \frac{v(t)}{t} d t
$$

Пусть $\lambda(r)$ - уточненный порядок такой, что

$$
\frac{d v(r)}{d \ln r} \uparrow+\infty, \quad \frac{d \ln v(r)}{d \ln r} \leqslant \frac{d \ln v_{0}(r)}{d \ln r} .
$$

Следующая теорема дает достаточное условие существования угловой плотности корней целых функций нулевого порядка, уточненньй порядок $\lambda(r)$ считающей функции корней которой удовлетворяет условию (2).

ТЕОРемА. Пусть $f-$ целая функиия нулевого порядка, $\lambda(r)$ - уточненный порядок функиии $n(r)$, удовлетворяющий условию (2). Если для почти всех $\theta, 0 \leqslant \theta<2 \pi$, существует равномерный относительно $\theta$ предел

$$
\lim _{r \rightarrow \infty} \frac{\ln f\left(r e^{i \theta}\right)-N(r)}{v(r)}
$$

$u$

$$
\lim _{r \rightarrow \infty} \frac{N(r)}{v_{0}(r)}=K, \quad 0<K<+\infty,
$$

то корни $f$ имеют угловую плотность.

При доказательстве этой теоремы будем использовать следующие утверждения. 
Лемма 1 [6]. Пусть $f$ - челая функиия, $f(0)=1, a_{j}-\kappa о р н и ~ f, \alpha_{j}=\arg a_{j}$. Обозначим

$$
\begin{gathered}
l_{k}(r, f)=\frac{1}{2 \pi} \int_{0}^{2 \pi} e^{-i k \theta} \ln f\left(r e^{i \theta}\right) d \theta, \quad k \in \mathbb{Z}, \\
n_{k}(r, f)=\sum_{\left|a_{j}\right| \leqslant r} e^{-i k \alpha_{j}}, \quad N_{k}(r, f)=\int_{0}^{r} \frac{n_{k}(t, f)}{t} d t, \quad k \in \mathbb{Z} .
\end{gathered}
$$

Тогда

$$
N_{k}(r, f)=l_{k}(r, f)-k \int_{0}^{r} \frac{l_{k}(t, f)}{t} d t, \quad k \in \mathbb{Z} .
$$

Мы будем использовать широко известную теорему Каратеодори-Леви (см., например, $[7$, с. 98]), которую сформулируем в виде следующей леммы.

Лемма 2. Пусть $\left(S_{n}\right)$ - последовательность мер на единичной окружности, которую мы будем отождествлять с последовательностью неубываюших непрерывных справа на $\mathbb{R}$ функиий, $S_{n}(x+2 \pi)-S_{n}(x)=S_{n}(2 \pi)-S_{n}(0), x \in \mathbb{R}$. Положим

$$
\Delta_{k}^{n}=\frac{1}{2 \pi} \int_{0}^{2 \pi} e^{-i k x} d S_{n}(x), \quad k \in \mathbb{Z} .
$$

Последовательность мер $\left(S_{n}\right)$ на единичной окружности сходится тогда и только тогда, когда для всех $k \in \mathbb{Z}$ существует конечный предел

$$
\lim _{n \rightarrow \infty} \Delta_{k}^{n}=\Delta_{k}
$$

Из теорем 5 и 6 работы $[8]$ вытекает следуюшая

Лемма 3. Пусть $g$ - непрерывно дифферениируемая выпуклая на $[a, b)$ функиия такая, что $g^{\prime}(x) \rightarrow+\infty$ при $x \rightarrow b u \ln g(x)$ вогнутая. Тогда для произвольной выпуклой функиии $f$ из существования предела

$$
\lim _{x \rightarrow b} \frac{f(x)}{g(x)}=K, \quad 0<K<+\infty,
$$

вытекает существование предела

$$
\lim _{x \rightarrow b} \frac{f^{\prime}(x)}{g^{\prime}(x)}=K
$$

ДОКАЗАТЕЛЬСТВо ТЕОРЕМЫ. Из определения коэффициентов $\Phi$ урье $l_{k}(r, f)$ и условий теоремы для $k \in \mathbb{Z}, k \neq 0$, имеем

$$
\frac{1}{2 \pi} \int_{0}^{2 \pi} \frac{\ln f\left(r e^{i \theta}\right)-N(r)}{v(r)} e^{-i k \theta} d \theta=\frac{l_{k}(r, f)}{v(r)}
$$

и, следовательно, существует предел

$$
\lim _{r \rightarrow \infty}{ }^{*} \frac{l_{k}(r, f)}{v(r)}=\frac{1}{2 \pi} \int_{0}^{2 \pi} H_{f}(\theta) e^{-i k \theta} d \theta,
$$


где $H_{f}(\theta)$ - функция из определения СРР. Так как $\left[7\right.$, c. 89] $l_{0}(r, f)=N(r)$, из $(3)$ получаем существование предела

$$
\lim _{r \rightarrow \infty} \frac{l_{0}(r, f)}{v_{0}(r)}
$$

Из теоремы Лопиталя следует, что

$$
\lim _{r \rightarrow \infty} * \frac{1}{v_{0}(r)} \int_{0}^{r} \frac{l_{k}(t, f)}{t} d t=\lim _{r \rightarrow \infty} * \frac{l_{k}(r, f)}{v(r)} .
$$

По лемме 1 имеем

$$
\frac{N_{k}(t, f)}{v_{0}(r)}=\frac{l_{k}(r, f)}{v_{0}(r)}-\frac{k}{v_{0}(r)} \int_{0}^{r} \frac{l_{k}(t, f)}{t} d t .
$$

Учитьвая, что $v(r) / v_{0}(r)=r v^{\prime}(r) / v(r) \rightarrow 0, r \rightarrow \infty$ (так как функция $v(r)$ медленно возрастающая), из (4) и (5) получаем

$$
\lim _{r \rightarrow \infty} \frac{N_{k}(t, f)}{v_{0}(r)}=-k \lim _{r \rightarrow \infty}^{*} \frac{1}{v_{0}(r)} \int_{0}^{r} \frac{l_{k}(t, f)}{t} d t .
$$

Используя рассуждения из [7, с. 143-144], имеем, что для всех $k \in \mathbb{Z}$ существуют пределы

$$
\lim _{r \rightarrow \infty} \frac{N_{k}(t, f)}{v_{0}(r)} .
$$

Положим $\alpha_{\nu}^{(m)}=\alpha_{\nu}+2 \pi m, m \in \mathbb{Z}, \alpha_{\nu}^{(0)}=\alpha_{\nu}$, где $\alpha_{\nu}=\arg a_{\nu}, a_{\nu}-$ корни $f$. Равенства

$$
\begin{gathered}
s(r, \varphi)-s(r, a)=2 \pi \sum_{a<\alpha_{\nu}^{(m)} \leqslant \varphi,\left|a_{\nu}\right| \leqslant r} 1, \\
s(r, \varphi+2 \pi)-s(r, \varphi)=s(r, a+2 \pi)-s(r, a)
\end{gathered}
$$

определяют при всевозможных наборах чисел $a \in \mathbb{R}$ и значений $s(r, a)$ семейство мер $(s(r, \varphi))$ на единичной окружности. Обозначим

$$
S(r, \varphi)-S(r, a)=\int_{0}^{r} \frac{s(t, \varphi)-s(t, a)}{t} d t .
$$

Тогда для каждого $\varphi_{0} \in \mathbb{R}$ верны равенства [7, с. 105]

$$
\begin{gathered}
S\left(r, \varphi_{0}+2 \pi\right)-S\left(r, \varphi_{0}\right)=S(r, a+2 \pi)-S(r, a)=2 \pi N(r), \\
n_{k}(r, f)=\frac{1}{2 \pi} \int_{\varphi_{0}}^{\varphi_{0}+2 \pi} e^{-i k \varphi} d s(r, \varphi), \\
N_{k}(r, f)=\frac{1}{2 \pi} \int_{\varphi_{0}}^{\varphi_{0}+2 \pi} e^{-i k \varphi} d S(r, \varphi) .
\end{gathered}
$$

По теореме Каратеодори-Леви из (6) и (8) получаем, что последовательность мер $S_{n}=$ $S\left(r_{n}, \varphi\right) / v_{0}\left(r_{n}\right)$, где $\left(r_{n}\right)$ - произвольная последовательность положительных чисел, 
$r_{n} \rightarrow+\infty, n \rightarrow+\infty$, сходится на единичной окружности, т.е. для почти всех $\varphi$ и $a$ существуют пределы

$$
\lim _{r \rightarrow \infty} \frac{S(r, \varphi)-S(r, a)}{v_{0}(r)}
$$

Поскольку, учитывая (2),

$$
\frac{d^{2} \ln v_{0}(r)}{(d \ln r)^{2}}=\frac{d}{d \ln r} \frac{v(r)}{v_{0}(r)}=\frac{r v(r)}{v_{0}(r)}\left(\frac{v^{\prime}(r)}{v(r)}-\frac{v_{0}^{\prime}(r)}{v_{0}(r)}\right) \leqslant 0
$$

функция $\ln v_{0}(r)$ является вогнутой относительно логарифма. Так как функции $S(r, \varphi)-S(r, a)$ и $v_{0}(r)$ вьпуклые относительно логарифма, из (9) и леммы 3 получаем, что для всех $k \in \mathbb{Z}$ существуют пределы

$$
\lim _{r \rightarrow \infty} \frac{n_{k}(r, f)}{v(r)}
$$

Используя еще раз теорему Каратеодори-Леви, из (7) и (10) имеем, что почти для всех $\varphi$ и $а$ существует предел

$$
\lim _{r \rightarrow \infty} \frac{s(r, \varphi)-s(r, a)}{v(r)}
$$

что доказьвает теорему.

\section{СПИСОК ЦИТИРОВАННОЙ ЛИТЕРАТУРЫ}

[1] Левин Б. Я. Распределение корней целых функций. М.: ГИТТЛ, 1956.

[2] Гольдберг А.А., Островский И.В. О производных и первообразных целых функций вполне регулярного роста // Теория функций, функцион. анализ и их прилож. 1973. № 18. C. $70-81$.

[3] Гольдберг А. А., Заболоцкий Н. В. Индекс концентрации субгармонической функции нулевого порядка // Матем. заметки. 1983. Т. 34. № 2. С. 227-236.

[4] Заболоцкий Н. В. Сильно регулярный рост целых функций нулевого порядка // Матем. заметки. 1998. Т. 63. № 2. С. 196-208.

[5] Zabolotskii N. V. An example of an entire function of strongly regular growth // Math. Stud. 2000. V. 13. № 2. P. 145-148.

[6] Калинець Р. З., Кондратюк А. А. Про регулярність зростання модуля і аргумента цілої функції в $L_{[0,2 \pi]}^{p}$-метриці // Укр. матем. ж. 1998. Т. 50. № 7. С. 889-896.

[7] Кондратюк А. А. Ряды Фурье и мероморфные функции. Львов: Вища школа, 1988.

[8] Братищев А. В. Об обращении правила Лопиталя // Механика сплошной среды. Ростов-на-Дону: Изд-во Рост. гос. ун-та, 1985. С. 28-42. 